Diabetologia (1994) 37: 3-9

\title{
Insulin-induced translocation of GLUT 4 in skeletal muscle of insulin-resistant Zucker rats
}

\author{
P. Galante ${ }^{1}$, E. Maerker ${ }^{1}$, R. Scholz ${ }^{1}$, K. Rett ${ }^{1}$, L.Herberg ${ }^{2}$, L. Mosthaf ${ }^{1}$, H. U.Häring ${ }^{1}$ \\ ${ }^{1}$ Institute for Diabetes Research, Munich, Germany \\ ${ }^{2}$ Diabetes Research Institute, Düsseldorf, Germany
}

Summary The genetically obese Zucker rat (fa/fa) is an animal model with severe insulin resistance of the skeletal muscle. We investigated whether a defect of insulindependent glucose transporter(GLUT 4) translocation might contribute to the pathogenesis of the insulin-resistant state. fa/fa rats, lean controls $(\mathrm{Fa} / \mathrm{Fa})$ as well as normal Wistar rats were injected intraperitoneally with insulin and were killed after 2 or $20 \mathrm{~min}$, respectively. Subcellular fractions were prepared from hind-limb skeletal muscle and were characterized by determination of marker-enzyme activities and immunoblotting applying antibodies against $\alpha 1 \mathrm{Na}^{+} / \mathrm{K}^{+}$AT Pase. The relative amounts of GLUT 1 and GLUT 4 were determined in the fractions by immunoblotting with the respective antibodies. Insulin induced an approximately two-fold increase of GLUT 4 in a plasma membrane and transverse tubule enriched fraction and a decrease in the low density enriched membrane fraction in all three groups of rats. There was a high individual variation in GLUT 4 translocation efficiency within the groups. However, no statistically significant difference was noted between the groups. No effect of insulin was detectable on the distribution of GLUT 1 or $\alpha 1 \mathrm{Na}^{+} \mathrm{K}^{+}$ATPase. The data suggest that skeletal muscle insulin resistance of obese Zucker rats is not associated with a lack of GLUT 4 translocation. [Diabetologia (1994) 37:3-9]

Key words Zucker rats, skeletal muscle, insulin resistance, glucose transporter (GLUT 1 and GLUT 4), GLUT 4 translocation.
The genetically obese Zucker rat (fa/fa) is an animal model characterized by extreme obesity, insulin resistance, hyperinsulinaemia, hyperlipidaemia and variable degrees of glucose intolerance [1-5]. This syndrome of the Zucker rat therefore shares several central characteristics with the insulin resistance syndrome described in humans [6-8]. The major site of insulin-mediated glucose disposal is in the skeletal muscle. Its insulin sensitivity is a major determinant for whole body insulin sensitivity. A severe insulin resistance of glucose uptake in the skeletal muscle has been described in fa/fa rats [9] and it is likely that this plays a pivotal role in the pathogenesis of the syndrome in these animals. Defects in glucose transport of the skeletal muscle of Zucker

Received: 22 February 1993

and in revised form: 1 July 1993

Corresponding author: Dr. H. U.Häring, Institute for Diabetes Research, Kölner Platz 1, D-80804 Munich, Germany and diabetic rats comprise a reduction in the basal rate as well as a reduced response to insulin stimulation [1013]. The underlying mechanism is unclear. We have earlier described that the kinase activity of insulin receptors isolated from skeletal muscle of Zucker rats is reduced [14]. This observation, which was recently confirmed by others [15] suggests that impaired signalling of the receptor might contribute to the reduced insulin response of the glucose transport system. Furthermore it is conceivable that defects in the glucose transport system itself might be responsible for the reduced response to insulin. The glucose transporter isoform which specifically mediates the insulin effect is GLUT 4 [16-17]. While a reduced content of GLUT 4 was reported for adipocytes of Zucker rats it appears that GLUT 4 levels in the skeletal muscle of Zucker rats are normal [18-19]. The stimulation of glucose transport by insulin in adipocytes and skeletal muscle involves two steps i.e. a translocation of GLUT 4 to cell surface membranes (plasma membrane (PM) and 
Table 1. Characteristics of the animals studied

\begin{tabular}{lccc}
\hline & $\begin{array}{l}\text { Wistar } \\
\text { rats i.p. }\end{array}$ & $\begin{array}{l}\text { Lean } \\
\text { Zucker } \\
\text { rats i.p. }\end{array}$ & $\begin{array}{l}\text { Obese } \\
\text { Zucker } \\
\text { rats i.p. }\end{array}$ \\
\hline $\begin{array}{l}\text { Age } \\
{[\text { weeks] }}\end{array}$ & 11 & $12-16$ & $11-21$ \\
$\begin{array}{l}\text { Body weight } \\
{[\mathrm{g}]}\end{array}$ & 311 & 315 & 496 \\
$\begin{array}{l}\text { Serum glucose before } \\
\text { insulin [mmol/1] }\end{array}$ & $6 \pm 1$ & $6.8 \pm 0.7$ & $10 \pm 3.3$ \\
$\begin{array}{l}\text { Serum glucose after } \\
\text { insulin [mmol/1] }\end{array}$ & $4.8 \pm 0.9$ & $3.5 \pm 0.4$ & $8.9 \pm 4.4$ \\
$\begin{array}{l}\text { Fasting insulin levels } \\
{[\mu U / m l] \text { ELISA }}\end{array}$ & $6.8 \pm 1.6$ & $7.5 \pm 1.4$ & $26.7 \pm 14.8$ \\
\hline
\end{tabular}

transverse tubules (TT)) as well as an activation of glucose transporters [20-22]. The aim of our study was to investigate whether an abnormality of GLUT 4 translocation might contribute to the pathogenesis of the insulin-resistant state of the skeletal muscle glucose transport system in Zucker rats.

\section{Materials and methods}

\section{Materials}

Aprotinin, benzamidine, vanadate, bacitracin, sodium fluoride, sodiumorthophosphate and phenylmethylsulphonyl fluoride (PMSF) were obtained from Sigma (Munich, FRG). All reagents for SDS-polyacrylamide gel electrophoresis were from Bio-Rad (Munich, FRG). All other reagents were of the best grade commercially available. Insulin (human) was purchased from Hoechst (Frankfurt, FRG). For homogenisation we used a dismembrator (mechanical device to grind tissue; Braun-Melsungen, FRG). For autoradiography X-Omat AR5 diagnostic film was used from Kodak (Rochester, New York, USA). Wistar rats were purchased from Savo (Kisslegg, FRG), lean and obese Zucker rats were a gift of Prof. L.Herberg (Düsseldorf, FRG). Animal characteristics are shown in Table 1 . The polyclonal sera against the GLUT 1 and GLUT 4 were obtained from WAKChemie (Homburg, FRG). The polyclonal $\alpha 1 \mathrm{Na}^{+} / \mathrm{K}^{+}$ATPase serum was from UBI (Lake Placid, N. Y., USA). Nitrocellulose was from Schleicher \& Schuell (Dassel, FRG) and the chemiluminescence detection system (ECL) was from Amersham (Braunschweig, FRG).

\section{Treatment of animals and hindlimb preparation}

The body weights of the Wistar rats ranged from $305-314 \mathrm{~g}$, of lean Zucker rats from 298-345 $\mathrm{g}$ and of obese Zucker rats from 344-672 g. Wistar rats, lean and obese Zucker rats were injected with insulin intraperitoneally or through the portal vein (insulin doses were adjusted to effect as described in the Results section). Subsequently the blood glucose concentration was determined every 5 min with Glucostix (Bayer, Leverkusen, FRG). The rats were killed $20 \mathrm{~min}$ after the insulin injection. The muscles of both hindlimbs were quickly removed, dissected on ice, and then deep-frozen at $-80^{\circ} \mathrm{C}$ within 3 min after the killing.
Muscle homogenate in TES-buffer

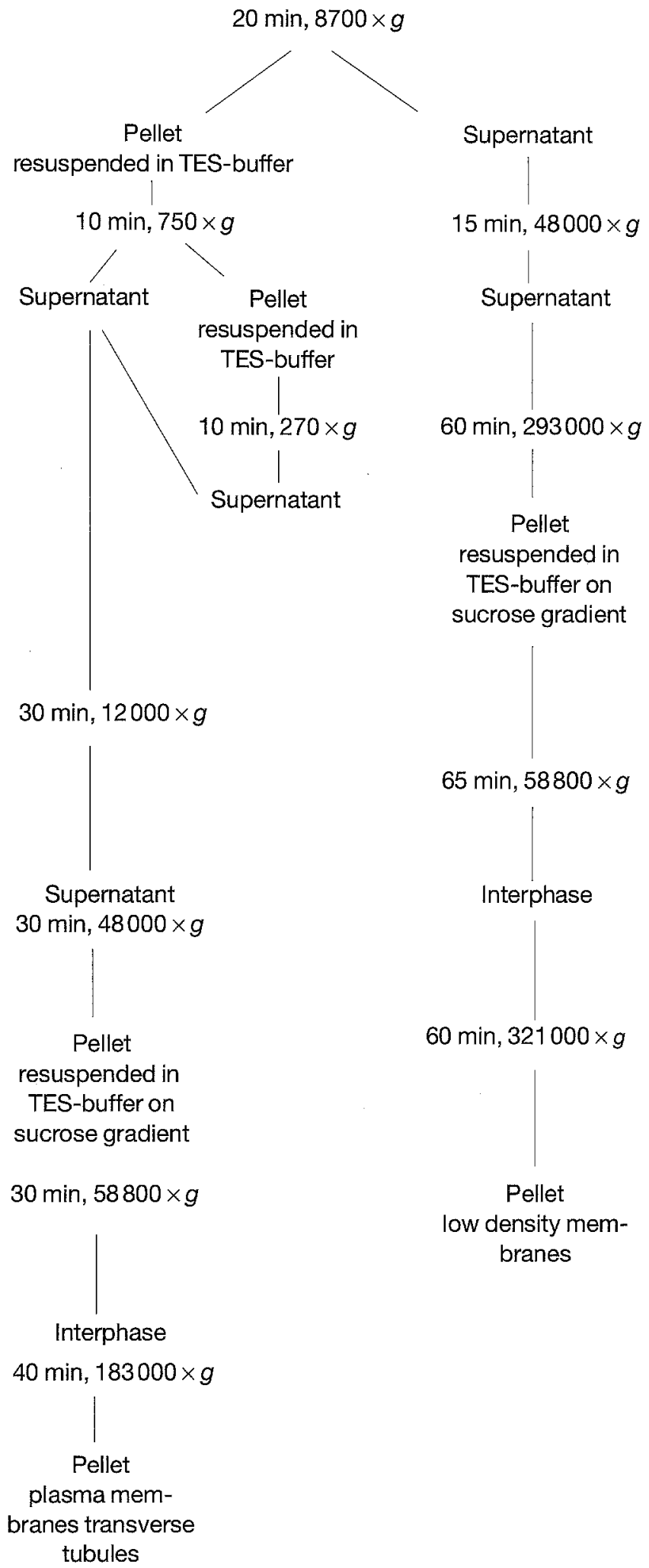

Fig. 1. Membrane preparation procedure 


\section{Membrane preparation and characterization}

For the preparation of membrane fractions enriched in PM and TT or low density membranes (LDM) we used the protocol which is described in Figure 1. The essential steps of the purification procedure were as follows: frozen muscles were homogenized with a dismembrator (15 mm Amplitude, full speed for $60 \mathrm{~s}$ ). Approximately $3 \mathrm{~g}$ of tissue was used in each group for the preparation. The homogenate was dissolved in cold $\left(4^{\circ} \mathrm{C}\right)$ buffer $(0.2 \mathrm{~mol} / 1 \mathrm{Tris} / \mathrm{HCl}, 1 \mathrm{mmol} / 1 \mathrm{EDTA}, 255 \mathrm{mmol} / 1$ sucrose, $\mathrm{pH} 7.4$ ) containing the following protease and phosphatase inhibitors: $0.5 \mathrm{mg} / \mathrm{ml}$ bacitracin, $1 \mathrm{mmol} / 1 \mathrm{PMSF}, 0.2 \mathrm{mg} / \mathrm{ml}$ benzamidine, $100 \mathrm{mmol} / 1$ sodium fluoride, $10 \mathrm{mmol} / 1$ sodium pyrophosphate, $1 \mathrm{mmol} / 1$ orthovanadate, and pottered in a glass/teflon potter 10 times. After homogenization the tissue was centrifuged at $8700 \times g$ for $20 \mathrm{~min}$. The supernatant consisting of fractions enriched with high density membranes (HDM) and LDM was centrifuged for $15 \mathrm{~min}$ at $48,000 \times g$ to separate the LDM-enriched membranes from the HDM-enriched membranes. To obtain the crude membrane pellet the supernatant was again centrifuged in a high-speed centrifuge for $60 \mathrm{~min}$ at $293,000 \times g$.

The pellet obtained with the first centrifugation was resuspended in buffer, pottered again and centrifuged at $750 \times \mathrm{g}$ for $10 \mathrm{~min}$. The resulting PM- and TT-enriched supernatant was centrifuged again for $30 \mathrm{~min}$ at $12,000 \times \mathrm{g}$. To obtain the crude membrane pellet the supernatant was centifuged for $30 \mathrm{~min}$ at $48,000 \times g$. Both pellets (LDM and PM + TT) were resuspended in buffer, pottered and layered on a $38.3 \%$ sucrose gradient. The PM- and TT-enriched fraction was centrifuged in an ultracentrifuge at $58,800 \times \mathrm{g}$ for $30 \mathrm{~min}$. The LDM-enriched fraction was centrifuged for $65 \mathrm{~min}$ at $58,800 \times g$. Both membrane pellets were then collected at the respective sucrose interphase, diluted four-fold with buffer and dispersed by mechanical disruption through a syringe needle. Finally the PM- and TT-enriched fraction was centrifuged for $40 \mathrm{~min}$ at $183,000 \times \mathrm{g}$. The LDM-enriched fraction was centrifuged for $60 \mathrm{~min}$ at $321,000 \times \mathrm{g}$. The entire preparation procedure was performed on ice. Marker enzymes were measured to determine the relative purity of the membrane fractions. $\mathrm{Na}^{+} / \mathrm{K}^{+}$ATPase served as a marker for $\mathrm{PM}$ and was determined by immunoblotting. As a marker for TT the $\mathrm{Mg}^{++}$-ATPase was determined as described previously [23]. To further ensure the membrane purity the NADH-cytochrome $\mathrm{C}$ reductase was measured [24-26]. Protein concentration was determined by Bio-Rad assay.

\section{Detection of GLUT 4 and GLUT 1 by immunoblotting}

Membranes $(10 \mu \mathrm{g}$ protein) were subjected to $10 \%$ SDS-PAGE [27] in the presence of $100 \mathrm{mmol} / 1$ DTT. Proteins were transferred to nitrocellulose by electroblotting (buffer: $192 \mathrm{mmol} / \mathrm{l}$

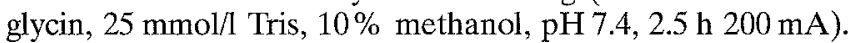
Following transfer the filters were blocked with $5 \%$ non-fat dry milk in NET buffer $(150 \mathrm{mmol} / 1 \mathrm{NaCl}, 5 \mathrm{mmol} / \mathrm{l}$ EDTA, $50 \mathrm{mmol} / 1$ Tris $\mathrm{pH} 7.4,0.05 \%$ Triton-X-100, $0.25 \%$ gelatine) for $1 \mathbf{h}$ at room temperature and subsequently incubated with the first antibody (GLUT 4 or GLUT 1 dilution in NET buffer) overnight at $4^{\circ} \mathrm{C}$. After incubation with the specific antibody, nitrocellulose was washed several times with $2 \%$ non-fat dry milk in NET buffer. Horseradish Peroxidase labelled IgG was then bound to the immunocomplexes. Visualization of the immunolabelled bands was carried out by autoradiography after addition of a chemiluminescence reagent (ECL).
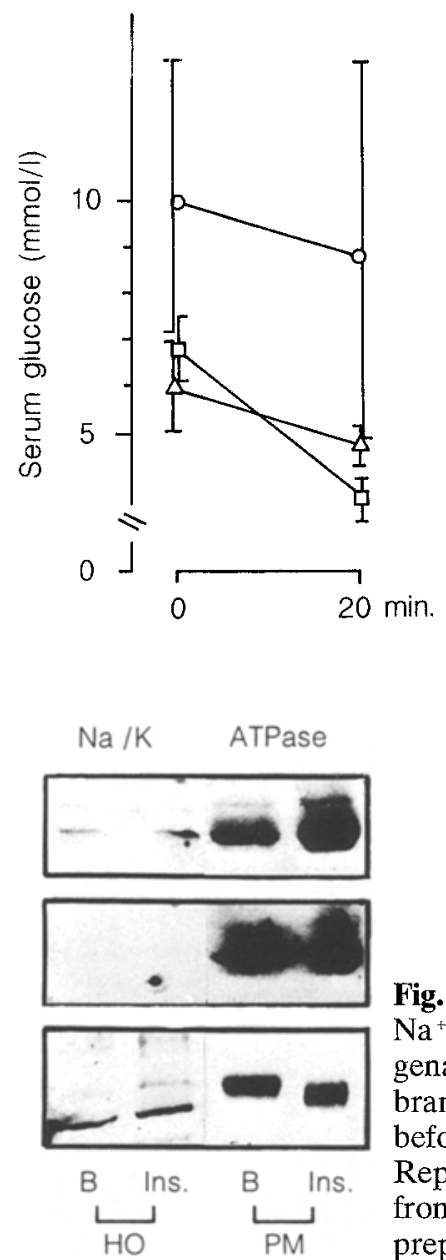

Fig.2. Effect of insulin on blood glucose levels in Wistar rats $(n=4$ pairs $)$ $\triangle$, lean Zucker rats $(n=$ 6 pairs) $\square$, obese Zucker rats $(n=8$ pairs $) \bigcirc$. Values are mean \pm SEM
Fig.3. Autoradiogram of $\alpha 1$ $\mathrm{Na}^{+} / \mathrm{K}^{+}$ATPase in crude homogenate $(\mathrm{HO})$ and plasma membrane (PM)-enriched fractions before and after insulin injection. Representative autoradiograms from three different membrane preparations are shown

\section{Scanning densitometry}

After autoradiography the density of the labelled $45 \mathrm{kDa}$ bands was measured using a type Elscript 400 scanner from Hirschmann $\mathrm{GmbH}$ (Munich, FRG). Measurements were performed with a $0.2 \mathrm{~mm}$ window in an extinction range of 2 or 4 by $100 \mu \mathrm{m}$ steps.

\section{Statistical analysis}

The data are expressed as mean values and $\mathrm{SD}$.

\section{Results}

In pilot experiments we empirically determined the amount of insulin which is necessary to induce a comparable decline of blood glucose in Wistar rats, lean Zucker rats and obese Zucker rats (5-15 IU insulin, depending on the body weight of the animals). The resulting decrease of blood glucose is shown in Figure 2. All animals were killed 20 min after insulin injection.

A flow sheet of the experimental protocol for subcellular fractionation is shown in Figure 1. The method is a procedure which we had used in an earlier study with spontaneous hypertensive rats (SHR) [28]. 


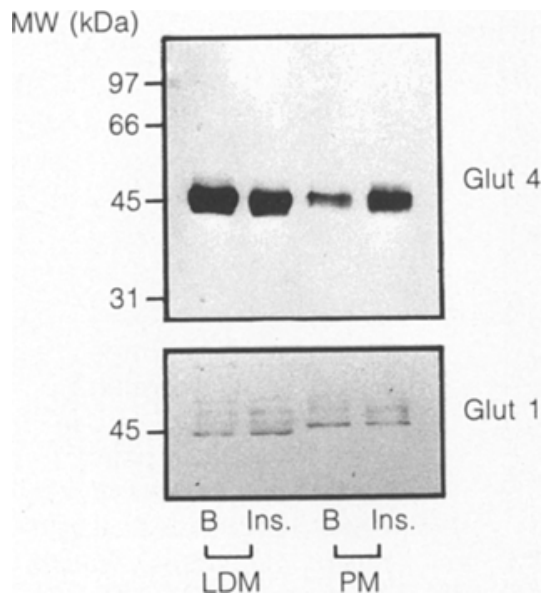

Fig.4. Autoradiogram of GLUT 4 and GLUT 1 in plasma membrane (PM) and low density membrane (LDM) fractions of skeletal muscle from Wistar rats. Membrane fractions were prepared as described in the Methods section and in Figure 1. Membrane proteins were separated by SDS-PAGE and transferred to nitrocellulose. The autoradiogram shows a representative immunoblot with antibodies directed against GLUT 4 and GLUT 1, respectively. The values were quantified by scanning densitometry. Insulin induced an increase of GLUT 4 labelling in the PM-fractions by $2.9 \pm 1.8$ SEM fold ( $n=4$ pairs) and a decrease of GLUT 4 labelling in the LDMfractions by $1.3 \pm 0.2$ SEM fold ( $n=3$ pairs)

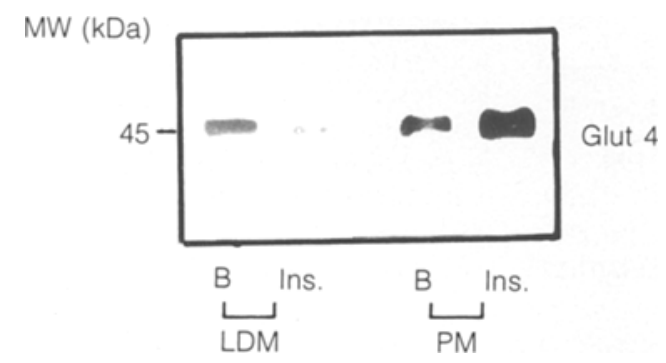

Fig.5. Autoradiogram of GLUT 4 in plasma membrane (PM) and low density membrane (LDM) fractions of skeletal muscle from lean Zucker rats. The experiment was performed as described in Figure 4. Insulin induced an increase of GLUT 4 labelling in the PMfraction by $2 \pm 1.7$ SEM fold ( $n=6$ pairs) and a decrease of GLUT 4 labelling in the LDM-fraction by $1.9 \pm 1.3 \mathrm{SEM}$ fold ( $n=6$ pairs)

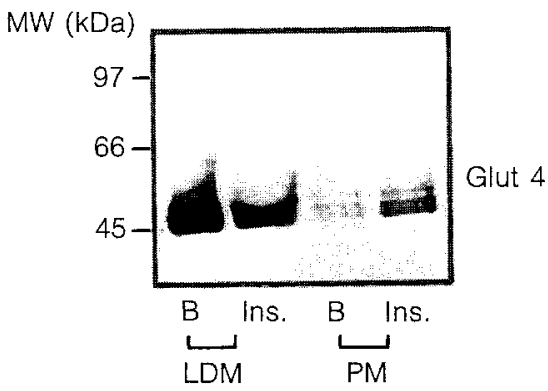

Fig.6. Autoradiogram of GLUT 4 in plasma membrane (PM) and low density membrane (LDM) fractions of skeletal muscle from obese Zucker rats. The experiment was performed as described in Figure 4. Insulin induced an increase of GLUT 4 labelling in the PM fraction by $2.3 \pm 1.7$ SEM fold ( $n=6$ pairs) and a decrease of GLUT 4 labelling in the LDM-fraction by $1.6 \pm 0.7 \mathrm{SEM}$ fold $(n=9$ pairs $)$
Using this protocol we previously obtained a $15-$ 18 fold enrichment of the ouabain sensitive $\mathrm{Na}^{+} / \mathrm{K}^{+}$ ATPase activity (enzyme assay), which is a marker for PM. In the present study the enrichment of $\mathrm{Na}^{+} / \mathrm{K}^{+}$ ATPase was determined by immunoblots using antibodies against $\alpha 1 \mathrm{Na}^{+} / \mathrm{K}^{+}$ATPase. We obtained a comparable enrichment of this marker protein in the different preparations (Fig.3). According to recent data published by Douen et al. [25] $\mathrm{Mg}^{++}$ATPase activity might be used as a marker for TT. The activity of this enzyme was $1.3 \pm 0.06 \mu \mathrm{mol} \cdot 1^{-1} \mathrm{Pi} \cdot \mathrm{mg}^{-1} \cdot \mathrm{min}^{-1}$ in the homogenate and $7.029 \pm 0.9 \mu \mathrm{mol} \cdot \mathrm{l}^{-1} \mathrm{Pi} \cdot \mathrm{mg}^{-1}$. $\mathrm{min}^{-1}$ in the PM + TT-enriched fraction. This suggests that the PM-fraction contains not only plasma membranes but is also enriched with transverse tubules (PM + TT-fraction).

The amount of GLUT 4 in the different membrane fractions of skeletal muscle was determined by immunoblots. Figure 4 shows a representative autoradiogram from Wistar rats which clearly demonstrates the insulin-induced increase of GLUT 4 in the PM- and TT-enriched fraction and the concomitant decrease in the LDM-enriched fraction. In the lower part of the figure the samples were immunoblotted with GLUT 1. In contrast to GLUT 4 no insulin-induced redistribution of GLUT 1 is detectable. The experiment was performed four times and the audioradiograms were quantified by scanning densitometry of the respective bands. The insulin-induced increase in the PM + TTfraction labelling of GLUT 4 was $2.9 \pm 1.8$ SEM fold ( $n=4$ pairs), the decrease in the LDM-fraction was $1.3 \pm 0.2$ SEM fold ( $n=3$ pairs).

The analogous experiment was performed with lean Zucker Rats. A representative autoradiogram is shown (Fig.5). As with the Wistar rats an insulin-induced increase of GLUT 4 in the PM + TT-fraction and a corresponding decrease in the LDM-fraction is visible. Again, there was no insulin effect detectable on the distribution of GLUT 1 in the respective membrane-fractions (data not shown). When all the experiments were quantified by scanning densitometry an insulin-induced increase of $2 \pm 1.7$ SEM fold ( $n=6$ pairs) and an insulin-induced decrease of $1.9 \pm 1.3 \operatorname{SEM}(n=6$ pairs $)$ was found. Due to the high individual variation in translocation efficiency and the relatively small number of animals used in the study ( $n=4$ pairs to $n=6$ pairs per group) we did not find statistically significant differences between Wistar rats and lean Zucker rats. However, we could not exclude that a larger study might reveal minor differences. While GLUT 4 was clearly translocated in all samples we could not detect any insulin effect on GLUT 1.

Figure 6 shows the effect of insulin on GLUT 4 labelling in PM + TT- and LDM-fractions of skeletalmuscle from obese Zucker rats. Again, the representative autoradiogram clearly exhibits an insulin-induced increase in the PM + TT-fractions and the corresponding decrease in the LDM-fraction. We quantified 


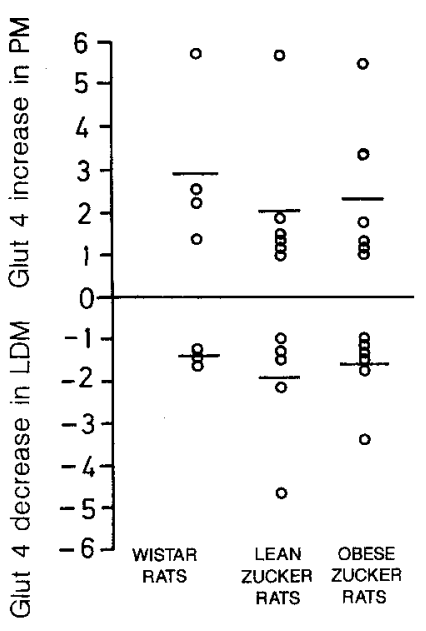

Fig. 7. Synopsis of the individual values of GLUT 4 increase in the plasma membranes (PM) and decrease in the low density membranes (LDM) of the animals studied

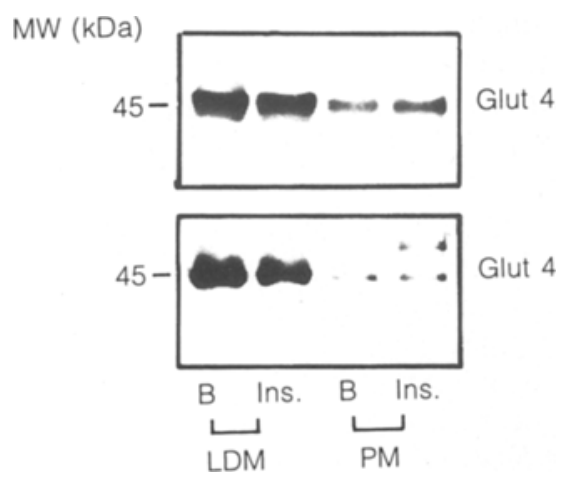

Fig. 8. Autoradiogram of GLUT 4 in plasma membrane (PM) and low density membrane (LDM) fractions of skeletal muscle from lean and obese Zucker rats. In contrast to previous experiments (Figs.4-6) animals were injected with insulin $(1 \mathrm{ml}$ of $10^{-5} \mathrm{mmol} / \mathrm{l}$ ) into the portal vein and killed $2 \mathrm{~min}$ after injection. Membrane fractions were prepared as described in the Methods section and in Figure 1. Membrane proteins were separated by SDS-PAGE and transferred to nitrocellulose and blotted with GLUT 4 and GLUT 1, respectively. The values were quantified by scanning densitometry. Insulin induced an increase of GLUT 4 labelling in the PM fraction by 1 fold (lean) or 1.6 fold (fa/fa) and a decrease of GLUT 4 labelling in the LDM fraction by 1.1 fold (lean) or 1.2 fold (fa/fa). The experiment was repeated once

LDM-fractions from ten pairs of animals by scanning densitometry and found a decrease of $1.6 \pm 0.7$ SEM fold in the LDM-fraction induced by insulin. PM + TTfractions were determined from 10 pairs of animals. There was an insulin-induced increase in the PM + TT labelling of $2.3 \pm 1.7 \mathrm{SEM}$ fold, as determined from the scanning data of six independent experiments. In four additional experiments we detected very efficent translocation of GLUT 4; however, we were not able to quantitate the data as fold increase of basal value since the basal levels were undetectable by scanning densitometry.

A synopsis of the individual values of all animals is shown in Figure 7. Although mean values between the groups slightly differed this was not statistically signifi- cant and could only be attributed to the relatively small number of animals included in the study. The data clearly show that after 20 min of insulin stimulation no significant difference was detectable between obese Zucker rats compared to lean Zucker rats or Wistar rats. There was a high individual variation within the three groups.

To search for possible differences in the kinetics of the insulin effects on GLUT 4 translocation we performed experiments applying short-term insulin stimulation. These experiments were done by insulin injection in the portal-vein followed by killing of the animal after $2 \mathrm{~min}$. Figure 8 shows an autoradiogram of membrane fractions obtained after 2 min of insulin stimulation. We then detected a slight decrease in LDM labelling and a slight increase in PM + TT labelling. This effect was observed both in lean and obese Zucker rats. Thus, even after short-term insulin stimulation we were unable to detect clear differences between insulin resistant and lean Zucker rats.

\section{Discussion}

Several studies have previously shown insulin resistance as well as a reduction of the basal rate of glucose transport in the skeletal muscle of Zucker and diabetic rats [10-13]. The present study was undertaken to assess whether this could be accounted for by a defect of the glucose transport system. GLUT 4 is the major insulin-regulatable glucose transporter. Since GLUT 4 expression levels are unaltered in the skeletal muscle of Zucker rats we decided to screen for defects in GLUT 4 transport activation at the level of translocation. Injection of the animals with insulin at doses which were shown to lower blood glucose levels, led to translocation of GLUT 4 from LDM to PM-fractions in the skeletal muscle of Wistar rats, lean Zucker rats and obese Zucker rats. Although mean values of translocation differed slightly between the three groups we do not consider this as significant due to large individual variations within the groups. We therefore conclude that there is no major defect in GLUT 4 translocation in fa/fa-rats. Several considerations led us to this interpretation of the data. The study compares GLUT 4 levels of insulin-injected animals with those of untreated animals. It is impossible to completely rule out that GLUT 4 content in internal or surface membranes might vary individually under basal conditions. However, in our hands the GLUT 1 content in the membrane fractions was very similar. GLUT 1 is not translocated upon insulin stimulation and was used as an internal protein standard. The autoradiograms showing GLUT 4 and GLUT 1 in parallel clearly demonstrate that the insulin-induced increase of GLUT 4 content in the plasma-membrane is, when compared to the amount of GLUT 1, clearly significant for all groups. Incorrect matching of membranes was excluded by 
parallel immunoblots with $\alpha 1 \mathrm{Na}^{+} / \mathrm{K}^{+}$ATPase antibodies. Another variable concerns the insulin concentrations finally reached in vivo. Insulin doses were matched according to their effect on blood glucose. Glucose levels were significantly lowered in all groups but always remained within the physiological range, none of the animals became hypoglycaemic. With the present experimental design it is impossible to establish dose-response curves of glucose transporter translocation. Therefore, we cannot exactly determine whether the individually observed effects on translocation are the result of maximal or only submaximal effective insulin concentrations. In this respect different individual kinetics of insulin action could influence the results. However, the short-term insulin stimulation experiments suggest that there is no major difference concerning the onset of the insulin effect. Whether this is also true for long-term kinetics is not clear. Considering these limitations of the experimental procedure the results of our study cannot be interpreted in a quantitative way. Subtle differences in kinetics or dose-response curves of GLUT 4 translocation, which we cannot measure with our assay, might be relevant for skeletal muscle insulin resistance of these animals. The data are, however, sufficiently controlled to conclude that obviously translocation of GLUT 4 is inducible in the skeletal muscle of fa/fa rats. This finding is in marked contrast to a recently published report [11]. The authors of the study were not able to detect GLUT 4 translocation in fatty Zucker rats. There are several possible explanations for the discrepancy of the results. The model of the Zucker rat might be more heterologous than has been considered so far. There might be subtypes in different strains with different mechanisms contributing to overall insulin resistance. Another variable that could influence the results is the composition of the membrane fractions. Marette et al. [26] have recently shown that translocation of GLUT 4 occurs both in the PM and in the TT-system [26]. While we obtained a 5.4-fold enrichement of TT-specific enzymes, King et al. [11] found a decrease (0.6-fold) in TT-specific enzymes. It is conceivable that the site of translocation is altered in the insulin-resistant animals. According to the marker enzymes the PM-fraction in our case contains a mixture of PM and TT. Despite similar insulininduced increases of GLUT 4 in this fraction there might be differences in the subcellular distribution between PM and TT between the groups. This will be important to clarify in the future. It is possible that in the study of King et al. [11] the authors were unable to detect translocation because their preparation contains another subset of membranes or just one type of membrane i.e. only PM.

In summary, we demonstrate here that insulin induces translocation of GLUT 4 in the skeletal muscle of insulin-resistant Zucker rats to a similar extent as in insulin-sensitive controls. We can therefore exclude the possibility of a complete lack of GLUT 4 translocation as a reason for the pathological alterations in Zucker rats, suggesting that other mechanisms are responsible for the insulin resistant state. However, future studies have to establish whether there is a difference in the targetting of GLUT 4 to PM or TT.

Acknowledgements. We thank the Sander-Stiftung and the Thyssen-Stiftung for support.

\section{References}

1. Bray CA (1977) The Zucker-fatty rat: a review. Fed Proc 36: 148-153

2. Crettaz M, Prentki M, Zaninetti D, Jeanrenaud B (1980) Insulin resistance in soleus muscle from obese Zucker rats. Biochem J 186: 525-534

3. Crettaz M, Zaninetti D, Jeanrenaud B (1981) Insulin resistance in heart and skeletal muscles of genetically obese Zucker rats. Biochem Soc Trans 9: 524-525

4. Czech MP, Richardson DK, Becker SG, Walters CG, Gitomer W, Heinrich J (1978) Insulin response in skeletal muscle and fat cells of the genetically obese Zucker rat. Metab Clin Exp 27 [Suppl 2]: 1967-1981

5. Kemmer FW, Berger M, Herberg L, Gries FA, Wirdeier A, Becker K (1979) Glucose metabolism in perfused skeletal muscle: demonstration of insulin resistance in obese Zucker rat. Biochem J 178: 733--741

6. Ferrannini E, Haffner SM, Mitchell BD, Stern MP (1991) Hyperinsulinaemia: the key feature of a cardiovascular and metabolic syndrome. Diabetologia 34: 416-422

7. Warram JH, Martin BH, Krolewski AS, Soeldner JS, Kahn CR (1990) Slow glucose removal rate and hyperinsulinemia precede the development of type 2 diabetes in the offspring of diabetic patients. Ann Intern Med 113: 909-915

8. Eriksson J, Franssila-Kallunki A, Ekstrand A et al. (1989) Early metabolic defects in persons at increased risk for noninsulin-dependent diabetes mellitus. N Engl J Med 321: $337-$ 343

9. Sherman WM, Katz AL, Cutler CL, Withers RT, Ivy JI (1988) Glucose transport: locus of muscle insulin resistance in obese Zucker rats. Am J Physiol 255: E374-E382

10. Kahn BB, Charrou MJ, Lodish MF, Cushman SW, Flier FS (1989) Differential regulation of two glucose transporters in adipose cells from diabetic and insulin-treated diabetic rats. $J$ Clin Invest 84: 404-411

11. King PA, Horton ED, Hirshman MF, Horton ES (1992) Insulin resistance in obese Zucker rat (fa/fa), skeletal muscle is associated with a failure of glucose transporter translocation. J Clin Invest 90: 1568-1575

12. Kahn BB, Rosetti L, Lodish HF, Charon MJ (1991) Decreased in vivo glucose uptake but normal expression of GLUT 1 and GLUT 4 in skeletal muscle of diabetic rats. J Clin Invest 87: 2197-2206

13. Kahn BB (1992) Facilitative glucose transporters: regulatory mechanism and dysregulation in diabetes. $\mathrm{J}$ Clin Invest 89 : $1367-1374$

14. Häring H, Obermaier-Kusser B (1989) Insulin receptor kinase defects in insulin-resistant tissues and their role in the pathogenesis of NIDDM. Diabetes/Metabolism Reviews 5: 431-441

15. Slieker LJ, Roberts EF, Shaw WW, Johnson WT (1990) Effect of streptozotocin-induced diabetes on insulin-receptor tyrosine kinase activity in obese Zucker rats. Diabetes 39: 619-625 
16. Burdett E, Beeler T, Klip A (1987) Distribution of glucose transporters and insulin receptors in the plasma membrane and transverse tubules of skeletal muscle. Arch Biochem Biophys 253: 279-286

17. Simpson IA, Cushman SW (1986) Hormonal regulation of mammalian glucose transport. Annu Rev Biochem 55: 1059 1089

18. Pedersen O, Kahn R, Kahn BB (1992) Divergent regulation of the GLUT 1 and GLUT 4 glucose transporters in isolated adipocytes from Zucker rats. J Clin Invest 89: 1964-1973

19. Klip A, Pâquet M (1990) Glucose transport and glucose transporters in muscle and their metabolic regulation. Diabetes Care 13: 228-243

20. Obermaier-Kusser B, Mühlbacher CH, Rattenhuber E et al. (1988) Further evidence for a two step model of glucose transport regulation. Biochem J 261: 699-705

21. Klip A, Ramlal T, Bilan PJ, Carter GD (1990) Recruitment of GLUT 4 glucose transporters by insulin in diabetic rat skeletal muscle. Biochem Biophys Res Commun 172: 728 736

22. Klip A, Ramlal T, Young DA, Hooloszy JO (1987) Insulin-induced translocation of glucose transporters in rat hindlimb muscle. FEBS letters 224: 224
23. Hidalgo C, Gonzales ME, Lagos R (1983) Characterization of the $\mathrm{Ca}$ - or $\mathrm{Mg}$-ATPase of transverse tubule membranes isolated from rabbit skeletal muscle. J Biol Chem 258: 13937 13945

24. Schimmel SD, Kent C, Bischoff R, Vagelos R (1973) Plasma membranes from cultured muscle cells: isolation procedure and separation of putative plasma-membrane marker enzymes. Proc Natl Acad Sci USA 70: 3195-3199

25. Douen AG, Burdett E, Ramlal T, Rastogi S, Vranic M, Klip A (1991) Characterization of glucose transporter-enriched membranes from rat skeletal muscle: assessment of endothelial cell contamination and presence of sarcoplasmic reticulum and transverse tubules. Endocrinology 128: 611-616

26. Marette A, Burdett E, Douen A, Vranic CM, Klip A (1992) Insulin induces the translocation of GLUT 4 from a unique intracellular organelle to transverse tubules in rat skeletal muscle. Diabetes 41: 1562-1569

27. Laemmli UK (1970) Cleavage of structural protein during the assembly of the head of bacteriophage $\mathrm{T}_{4}$. Nature (London) 227:680-685

28. Bader S, Scholz R, Kellerer Met al. (1992) Normal insulin receptor tyrosine kinase activity and glucose transporter (GLUT 4) levels in the skeletal muscle of hyperinsulinaemic hypertensive rats. Diabetologia 35: 712-718 